Journal of Applied AnALysis

Vol. 13, No. 1 (2007), pp. 117-132

\title{
SECOND ORDER MIXED SYMMETRIC DUALITY IN NON-DIFFERENTIABLE MULTI-OBJECTIVE MATHEMATICAL PROGRAMMING
}

\author{
S. K. MISHRA \\ Received November 8, 2005 and, in revised form, June 6, 2006
}

\begin{abstract}
A pair of Mond-Weir type second order mixed symmetric duals is presented for a class of non-differentiable multi-objective nonlinear programming problems with multiple arguments. We establish duality theorems for the new pair of dual models under second order generalized convexity assumptions. This mixed second order dual formulation unifies the two existing second order symmetric dual formulations in the literature. Many recent works on symmetric duality are obtained as special cases of the results established in the present paper.
\end{abstract}

\section{IntRoduction}

Dorn [7] introduced symmetric duality in nonlinear programming by defining a program and its dual to be symmetric if the dual of the dual

2000 Mathematics Subject Classification. Primary: 49N15, 90C11, 90C30, 90C46.

Key words and phrases. Second order symmetric duality, non-differentiable multiobjective programming, duality theorems, second order $F$-convexity, support function.

This research is supported by the Department of Science and Technology, Ministry of Science and Technology, Government of India, under the SERC, Fast Track Scheme for Young Scientists 2001-2002, through grant No. SR/FTP/MS-22/2001

ISSN 1425-6908 C Heldermann Verlag. 
is the original problem. The symmetric duality for scalar programming has been studied extensively in the literature, one can refer to Dantzig et al. [5], Mishra [14]-[16], Mond [18], Mond and Weir [21].

Mond and Schechter [20] studied non-differentiable symmetric duality for a class of optimization problems in which the objective function consists of support functions. Following Mond and Schechter [20], Chen [4], Hou and Yang [11], and Yang et al. [26], [28], studied symmetric duality for such problems.

The study of second order duality is significant due to the computational advantage over the first order duality as it provides tighter bounds for the value of the objective function when approximations are used (see [9], [13], [17], [19]). Mond [19] was the first one to present second order symmetric dual models and proved second order symmetric duality theorems under convexity assumptions. Mishra [15] established Wolfe type and Mond-Weir type second order symmetric duality for nonlinear programming problems under second order generalized convexity assumptions. Hou and Yang [11] and Yang et al. [26] extended the results of Mishra [15] to the non-differentiable case. Suneja et al. [24] extended the results of Mishra [15] to the multiobjective case. Some more results on symmetric duality can be seen in [6], [21], [22], [25].

Yang et al. [27] presented a mixed symmetric dual formulation for a nondifferentiable nonlinear programming problem. Bector et al. [3] introduced a mixed symmetric dual model for a class of nonlinear multiobjective programming problems. However, the models given by Bector et al. [3] as well as by Yang et al. [27] do not allow the further weakening of generalized convexity assumptions on a part of the objective functions.

Very recently, Yang et al. [28] established second order duality results for second order symmetric dual models for a class of non-differentiable multiobjective programming problems as an extension of the results of Suneja et al. [24].

In this paper, a pair of Mond-Weir type second order mixed symmetric dual is presented for a class of non-differentiable multi-objective nonlinear programming problems with multiple arguments. We establish duality theorems for the new pair of dual models under second order generalized convexity assumptions. This mixed second order dual formulation unifies the two existing second order symmetric dual formulations in the literature. Many recent works on symmetric duality are obtained as special cases of the results established in the present paper. 


\section{Preliminaries}

Let $\mathbb{R}^{n}$ denote the $n$-dimensional Euclidean space and let $\mathbb{R}_{+}^{n}$ be its nonnegative orthant. The following ordering relations for vectors in $\mathbb{R}^{n}$ will be used in this paper:

For $x, y \in \mathbb{R}^{n}$, by $x<y$ we mean $y-x \in \operatorname{int} \mathbb{R}_{+}^{n}$; by $x \leq y$ we mean $y-x \in \mathbb{R}_{+}^{n} \backslash\{0\}$; and by $x \leqq y$ we mean $y-x \in \mathbb{R}_{+}^{n}$. The negation of $x \leq y$ is denoted by $x \not \leq y$.

Let $f(x, y)$ be real valued twice differentiable function defined on $\mathbb{R}^{n} \times \mathbb{R}^{m}$. Let $\nabla_{x} f(\bar{x}, \bar{y})$ and $\nabla_{y} f(\bar{x}, \bar{y})$ denote the partial derivatives of $f(x, y)$ with respect to $x$ and $y$ at $(\bar{x}, \bar{y})$. Let $\nabla_{x x} f(\bar{x}, \bar{y})$ denote the $n \times n$ symmetric Hessian matrix at $(\bar{x}, \bar{y}), \nabla_{x y} f(\bar{x}, \bar{y}), \nabla_{y x} f(\bar{x}, \bar{y})$ and $\nabla_{y y} f(\bar{x}, \bar{y})$ are defined similarly. Consider the following multiobjective programming problem $(\mathrm{VP})$ :

$$
\operatorname{Min}\left(f_{1}(x), f_{2}(x), \ldots, f_{p}(x)\right) \quad \text { subject to } x \in X_{0},
$$

where $f_{i}: \mathbb{R}^{n} \rightarrow \mathbb{R}, i=1,2, \ldots, p$ and $X_{0} \subseteq \mathbb{R}^{n}$.

For problem (VP), an efficient solution and a properly efficient solution are defined as follows:

Definition 1. A feasible solution $x^{0}$ is said to be an efficient solution for (VP) if there exists no other feasible solution $x$ such that

$$
f(x) \leq f\left(x^{0}\right) .
$$

Definition 2. A feasible solution $x^{0}$ is said to be properly efficient solution for (VP) if it is efficient for (VP) and there exists a scalar $M>0$ such that, for each $i$, we have

$$
\frac{f_{i}\left(x^{0}\right)-f_{i}(x)}{f_{j}(x)-f_{j}\left(x^{0}\right)} \leqq M
$$

for some $j$ such that $f_{j}(x)>f_{j}\left(x^{0}\right)$ whenever $x \in X_{0}$ and $f_{i}(x)<f_{i}\left(x^{0}\right)$.

Let $C$ be a compact convex set in $\mathbb{R}^{n}$. The support function of $C$ is defined by

$$
s(x \mid C)=\max \left\{x^{T} y: y \in C\right\} .
$$

A support function, being convex and everywhere finite, has a subdifferential [23], that is, there exists $z \in \mathbb{R}^{n}$ such that

$$
s(y \mid C) \geqq s(x \mid C)+z^{T}(y-x), \quad \forall y \in C .
$$

The subdifferential of $s(x \mid C)$ is given by

$$
\partial s(x \mid C)=\left\{z \in C: z^{T} x=s(x \mid C)\right\} .
$$


For any set $D \subset \mathbb{R}^{n}$, the normal cone to $D$ at a point $x \in D$ is defined by

$$
N_{D}(x)=\left\{y \in \mathbb{R}^{n}: y^{T}(z-x) \leqq 0, \forall z \in D\right\} .
$$

It is obvious that for a compact convex set $C, y \in N_{C}(x)$ if and only if $s(y \mid C)=x^{T} y$, or equivalently, $x \in \partial s(y \mid C)$.

The following definitions will be needed in the sequel:

Definition 3. Let $X \subset \mathbb{R}^{n}$. A functional $F: X \times X \times \mathbb{R}^{n} \rightarrow \mathbb{R}$ is said to be sublinear with respect to its third argument if for any $x, y \in X$

(A) $F\left(x, y ; a_{1}+a_{2}\right)=F\left(x, y ; a_{1}\right)+F\left(x, y ; a_{2}\right)$ for any $a_{1}, a_{2} \in \mathbb{R}^{n}$;

(B) $F(x, y ; \alpha a)=\alpha F(x, y ; a)$ for any $\alpha \in \mathbb{R}_{+}$and $a \in \mathbb{R}^{n}$.

Definition 4. Let $X \subset \mathbb{R}^{n}, Y \subset \mathbb{R}^{m}$ and $F: X \times Y \times \mathbb{R}^{n} \rightarrow \mathbb{R}$ be sublinear with respect to its third argument. $f(\cdot, y)$ is said to be second order $F$ convex at $\bar{x} \in X$, with respect to $p \in \mathbb{R}^{n}$, for fixed $y \in Y$, if

$$
\begin{array}{r}
f(x, y)-f(\bar{x}, y)+\frac{1}{2} p^{T} \nabla_{x x} f(\bar{x}, y) p \geqq F\left(x, \bar{x} ; \nabla_{x} f(\bar{x}, y)+\nabla_{x x} f(\bar{x}, y) p\right), \\
\forall x \in X .
\end{array}
$$

$f$ is said to be second order $F$-concave at $\bar{x} \in X$, with respect to $p \in \mathbb{R}^{n}$, for fixed $y \in Y$, if $-f$ is second order $F$-convex at $\bar{x} \in X$, with respect to $p \in \mathbb{R}^{n}$.

Definition 5. Let $X \subset \mathbb{R}^{n}, Y \subset \mathbb{R}^{m}$ and $F: X \times Y \times \mathbb{R}^{n} \rightarrow \mathbb{R}$ be sublinear with respect to its third argument. $f(x, \cdot)$ is said to be second order $F$ pseudo-convex at $\bar{x} \in X$, with respect to $p \in \mathbb{R}^{n}$, for fixed $y \in Y$, if

$$
\begin{aligned}
& F\left(x, \bar{x} ; \nabla_{x} f(\bar{x}, y)+\nabla_{x x} f(\bar{x}, y) p\right) \geqq 0 \\
& \Rightarrow f(x, y) \geqq f(\bar{x}, y)-\frac{1}{2} p^{T} \nabla_{x x} f(\bar{x}, y) p, \quad \forall x \in X .
\end{aligned}
$$

$f$ is said to be second order $F$-pseudo-concave at $\bar{x} \in X$, with respect to $p \in \mathbb{R}^{n}$, for fixed $y \in Y$, if $-f$ is second order $F$-pseudo-convex at $\bar{x} \in X$, with respect to $p \in \mathbb{R}^{n}$.

\section{Remark 1.}

(i) The second order $F$-pseudo-convexity reduces to the $F$-pseudoconvexity introduced by Hanson and Mond [10] when $p=0$.

(ii) For $F(x, \bar{x} ; a)=\eta(x, \bar{x})^{T} a$, and $p=0$, where $\eta: X \times X \rightarrow \mathbb{R}^{n}$, the second order $F$-convexity reduces to the invexity introduced by Hanson [8], and second order $F$-pseudo-convexity reduces to the pseudoinvexity introduced by Hanson [8]. 


\section{Mixed type multi-objeCtive Symmetric DUALity}

For $N=\{1,2, \ldots, n\}$ and $M=\{1,2, \ldots, m\}$ let $J_{1} \subset N, K_{1} \subset M$ and $J_{2}=N \backslash J_{1}$ and $K_{2}=M \backslash K_{1}$. Let $\left|J_{1}\right|$ denote the number of elements in the set $J_{1}$. The numbers $\left|J_{2}\right|,\left|K_{1}\right|$ and $\left|K_{2}\right|$ are defined similarly. Notice that if $J_{1}=\Phi$, then $J_{2}=N$, that is, $\left|J_{1}\right|=0$ and $\left|J_{2}\right|=n$. Hence, $\mathbb{R}^{\left|J_{1}\right|}$ is zero dimensional Euclidean space and $\mathbb{R}^{\left|J_{2}\right|}$ is $n$-dimensional Euclidean space. It is clear that any $x \in \mathbb{R}^{n}$ can be written as $x=\left(x^{1}, x^{2}\right), x^{1} \in \mathbb{R}^{\left|J_{1}\right|}$, $x^{2} \in \mathbb{R}^{\left|J_{2}\right|}$. Similarly, any $y \in \mathbb{R}^{m}$ can be written as $y=\left(y^{1}, y^{2}\right), y^{1} \in \mathbb{R}^{\left|K_{1}\right|}$, $y^{2} \in \mathbb{R}^{\left|K_{2}\right|}$. Let $f: \mathbb{R}^{\left|J_{1}\right|} \times \mathbb{R}^{\left|K_{1}\right|} \rightarrow \mathbb{R}^{l}$ and $g: \mathbb{R}^{\left|J_{2}\right|} \times \mathbb{R}^{\left|K_{2}\right|} \rightarrow \mathbb{R}^{l}$ be twice differentiable functions and $e=(1,1, \ldots, 1)^{T} \in \mathbb{R}^{l}$.

Now we can introduce the following pair of non-differentiable multiobjective programs and discuss duality theorems under some mild assumptions of generalized convexity.

Primal Problem (SMP)

Min $H\left(x^{1}, x^{2}, y^{1}, y^{2}, z^{1}, z^{2}, p^{1}, p^{2}, \lambda\right)$

$$
=\left(H_{1}\left(x^{1}, x^{2}, y^{1}, y^{2}, z^{1}, z^{2}, p^{1}, p^{2}, \lambda\right), \ldots, H_{l}\left(x^{1}, x^{2}, y^{1}, y^{2}, z^{1}, z^{2}, p^{1}, p^{2}, \lambda\right)\right)
$$

subject to

$$
\begin{gathered}
\left(x^{1}, x^{2}, y^{1}, y^{2}, z^{1}, z^{2}, p^{1}, p^{2}, \lambda\right) \\
\in \mathbb{R}^{\left|J_{1}\right|} \times \mathbb{R}^{\left|J_{2}\right|} \times \mathbb{R}^{\left|K_{1}\right|} \times \mathbb{R}^{\left|K_{2}\right|} \times \mathbb{R}^{\left|K_{1}\right|} \times \mathbb{R}^{\left|K_{2}\right|} \times \mathbb{R}^{\left|K_{1}\right|} \times \mathbb{R}^{\left|K_{2}\right|} \times \mathbb{R}_{+}^{l} \\
\sum_{i=1}^{l} \lambda_{i}\left[\nabla_{y^{1}} f_{i}\left(x^{1}, y^{1}\right)-z_{i}^{1}+\nabla_{y^{1} y^{1}} f_{i}\left(x^{1}, y^{1}\right) p_{i}^{1}\right] \leqq 0 \\
\sum_{i=1}^{l} \lambda_{i}\left[\nabla_{y^{2}} g_{i}\left(x^{2}, y^{2}\right)-z_{i}^{2}+\nabla_{y^{2} y^{2}} g_{i}\left(x^{2}, y^{2}\right) p_{i}^{2}\right] \leqq 0 \\
\left(y^{1}\right)^{T} \sum_{i=1}^{l} \lambda_{i}\left[\nabla_{y^{1}} f_{i}\left(x^{1}, y^{1}\right)-z_{i}^{1}+\nabla_{y^{1} y^{1}} f_{i}\left(x^{1}, y^{1}\right) p_{i}^{1}\right] \geqq 0 \\
\left(y^{2}\right)^{T} \sum_{i=1}^{l} \lambda_{i}\left[\nabla_{y^{2}} g_{i}\left(x^{2}, y^{2}\right)-z_{i}^{2}+\nabla_{y^{2} y^{2}} g_{i}\left(x^{2}, y^{2}\right) p_{i}^{2}\right] \geqq 0 \\
\left(x^{1}, x^{2}\right) \geqq 0, \\
z_{i}^{1} \in D_{i}^{1}, \text { and } z_{i}^{2} \in D_{i}^{2}, i=1,2, \ldots, l \\
\lambda>0, \sum_{i=1}^{l} \lambda_{i}=1 .
\end{gathered}
$$


Dual Problem (SMD)

$\operatorname{Max} G\left(u^{1}, u^{2}, v^{1}, v^{2}, w^{1}, w^{2}, q^{1}, q^{2}, \lambda\right)$

$=\left(G_{1}\left(u^{1}, u^{2}, v^{1}, v^{2}, w^{1}, w^{2}, q^{1}, q^{2}, \lambda\right), \ldots, G_{l}\left(u^{1}, u^{2}, v^{1}, v^{2}, w^{1}, w^{2}, q^{1}, q^{2}, \lambda\right)\right)$ subject to

$$
\begin{gathered}
\left(u^{1}, u^{2}, v^{1}, v^{2}, w^{1}, w^{2}, q^{1}, q^{2}, \lambda\right) \\
\in \mathbb{R}^{\left|J_{1}\right|} \times \mathbb{R}^{\left|J_{2}\right|} \times \mathbb{R}^{\left|K_{1}\right|} \times \mathbb{R}^{\left|K_{2}\right|} \times \mathbb{R}^{\left|J_{1}\right|} \times \mathbb{R}^{\left|J_{2}\right|} \times \mathbb{R}^{\left|J_{1}\right|} \times \mathbb{R}^{\left|J_{2}\right|} \times \mathbb{R}_{+}^{l} \\
\sum_{i=1}^{l} \lambda_{i}\left[\nabla_{u^{1}} f_{i}\left(u^{1}, v^{1}\right)+w_{i}^{1}+\nabla_{u^{1} u^{1}} f_{i}\left(u^{1}, v^{1}\right) q_{i}^{1}\right] \geqq 0 \\
\sum_{i=1}^{l} \lambda_{i}\left[\nabla_{u^{2}} g_{i}\left(u^{2}, v^{2}\right)+w_{i}^{2}+\nabla_{u^{2} u^{2}} g_{i}\left(u^{2}, v^{2}\right) q_{i}^{2}\right] \geqq 0 \\
\left(u^{1}\right)^{T} \sum_{i=1}^{l} \lambda_{i}\left[\nabla_{u^{1}} f_{i}\left(u^{1}, v^{1}\right)+w_{i}^{1}+\nabla_{u^{1} u^{1}} f_{i}\left(u^{1}, v^{1}\right) q_{i}^{1}\right] \leqq 0 \\
\left(u^{2}\right)^{T} \sum_{i=1}^{l} \lambda_{i}\left[\nabla_{u^{2}} g_{i}\left(u^{2}, v^{2}\right)+w_{i}^{2}+\nabla_{u^{2} u^{2}} g_{i}\left(u^{2}, v^{2}\right) q_{i}^{2}\right] \leqq 0 \\
\left(v^{1}, v^{2}\right) \geqq 0, \\
w_{i}^{1} \in C_{i}^{1}, \text { and } w_{i}^{2} \in C_{i}^{2}, i=1,2, \ldots, l \\
\lambda>0, \sum_{i=1}^{l} \lambda_{i}=1
\end{gathered}
$$

where

$$
\begin{aligned}
& H_{i}\left(x^{1}, x^{2}, y^{1}, y^{2}, z^{1}, z^{2}, p^{1}, p^{2}, \lambda\right) \\
& =f_{i}\left(x^{1}, y^{1}\right)+g_{i}\left(x^{2}, y^{2}\right)+s\left(x^{1} \mid C_{i}^{1}\right)+s\left(x^{2} \mid C_{i}^{2}\right)-\left(y^{1}\right)^{T} z_{i}^{1} \\
& -\left(y^{2}\right)^{T} z_{i}^{2}-\frac{1}{2}\left(p_{i}^{1}\right)^{T} \nabla_{y^{1} y^{1}} f_{i}\left(x^{1}, y^{1}\right) p_{i}^{1}-\frac{1}{2}\left(q_{i}^{2}\right)^{T} \nabla_{y^{2} y^{2}} g_{i}\left(x^{2}, y^{2}\right) q_{i}^{2} \\
& G_{i}\left(u^{1}, u^{2}, v^{1}, v^{2}, w^{1}, w^{2}, q^{1}, q^{2}, \lambda\right) \\
& =f_{i}\left(u^{1}, v^{1}\right)+g_{i}\left(u^{2}, v^{2}\right)-s\left(v^{1} \mid D_{i}^{1}\right)-s\left(v^{2} \mid D_{i}^{2}\right)+\left(u^{1}\right)^{T} w_{i}^{1} \\
& +\left(u^{2}\right)^{T} w_{i}^{2}-\frac{1}{2}\left(q_{i}^{1}\right)^{T} \nabla_{u^{1} u^{1}} f_{i}\left(u^{1}, v^{1}\right) q_{i}^{1}-\frac{1}{2}\left(q_{i}^{2}\right)^{T} \nabla_{u^{2} u^{2}} g_{i}\left(u^{2}, v^{2}\right) q_{i}^{2}
\end{aligned}
$$

and $C_{i}^{1}$ is a compact and convex subsets of $\mathbb{R}^{\left|J_{1}\right|}$ for $i=1,2, \ldots, l$ and $C_{i}^{2}$ is a compact and convex subsets of $\mathbb{R}^{\left|J_{2}\right|}$ for $i=1,2, \ldots, l$, similarly, $D_{i}^{1}$ is a compact and convex subsets of $\mathbb{R}^{\left|K_{1}\right|}$ for $i=1,2, \ldots, l$ and $D_{i}^{2}$ is a compact and convex subsets of $\mathbb{R}^{\left|K_{2}\right|}$ for $i=1,2, \ldots, l$. 
For the first model, we can prove the following weak duality theorem.

Theorem 1 (Weak duality). Let $\left(x^{1}, x^{2}, y^{1}, y^{2}, z^{1}, z^{2}, p^{1}, p^{2}, \lambda\right)$ be feasible for $(S M P)$ and $\left(u^{1}, u^{2}, v^{1}, v^{2}, w^{1}, w^{2}, q^{1}, q^{2}, \lambda\right)$ be feasible for $(S M D)$. Suppose there exist sublinear functionals $F_{1}, F_{2}, G_{1}$ and $G_{2}$ satisfying the following conditions:

(i) $F_{1}\left(x^{1}, u^{1} ; a\right)+\left(u^{1}\right)^{T} a \geqq 0$ if $a \geqq 0$;

(ii) $G_{1}\left(x^{2}, u^{2} ; b\right)+\left(u^{2}\right)^{T} b \geqq 0$ if $b \geqq 0$;

(iii) $F_{2}\left(v^{1}, y^{1} ; c\right)+\left(y^{1}\right)^{T} c \geqq 0$ if $c \geqq 0$;

(iv) $G_{2}\left(v^{2}, y^{2} ; d\right)+\left(y^{2}\right)^{T} d \geqq 0$ if $d \geqq 0$.

Furthermore, assume that for $i=1,2, \ldots, l, f_{i}\left(\cdot, v^{1}\right)+\cdot{ }^{T} w_{i}^{1}$ is second order $F_{1}$-convex for fixed $v^{1}$, with respect to $q_{i}^{1} \in \mathbb{R}^{\left|J_{1}\right|}, f_{i}\left(x^{1}, \cdot\right)-{ }^{T} z_{i}^{1}$ is second order $F_{2}$-concave for fixed $x^{1}$, with respect to $p_{i}^{1} \in \mathbb{R}^{\left|K_{1}\right|}, g_{i}\left(\cdot, v^{2}\right)+{ }^{T} w_{i}^{2}$ is second order $G_{1}$-convex for fixed $v^{2}$ with respect to $q_{i}^{2} \in \mathbb{R}^{\left|J_{2}\right|}$ and $g_{i}\left(x^{2}, \cdot\right)$ ${ }^{T} z_{i}^{2}$ is second order $G_{2}$-concave for fixed $x^{2}$, with respect to $p_{i}^{2} \in \mathbb{R}^{\left|K_{2}\right|}$.

Then

$$
H\left(x^{1}, x^{2}, y^{1}, y^{2}, z^{1}, z^{2}, p^{1}, p^{2}, \lambda\right) \not \leq G\left(u^{1}, u^{2}, v^{1}, v^{2}, w^{1}, w^{2}, q^{1}, q^{2}, \lambda\right) .
$$

Proof. Assume that the result is not true, that is,

$$
H\left(x^{1}, x^{2}, y^{1}, y^{2}, z^{1}, z^{2}, p^{1}, p^{2}, \lambda\right) \leq G\left(u^{1}, u^{2}, v^{1}, v^{2}, w^{1}, w^{2}, q^{1}, q^{2}, \lambda\right) .
$$

Then, since $\lambda>0$, we have

$$
\begin{aligned}
& \sum_{i=1}^{l} \lambda_{i}\left[f_{i}\left(x^{1}, y^{1}\right)+g_{i}\left(x^{2}, y^{2}\right)+s\left(x^{1} \mid C_{i}^{1}\right)+s\left(x^{2} \mid C_{i}^{2}\right)-\left(y^{1}\right)^{T} z_{i}^{1}\right. \\
& -\left(y^{2}\right)^{T} z_{i}^{2}-\frac{1}{2}\left(p_{i}^{1}\right)^{T} \nabla_{y^{1} y^{1}} f_{i}\left(x^{1}, y^{1}\right) p_{i}^{1} \\
& \left.-\frac{1}{2}\left(p_{i}^{2}\right)^{T} \nabla_{y^{2} y^{2}} g_{i}\left(x^{2}, y^{2}\right) p_{i}^{2}\right] \\
& <\sum_{i=1}^{l} \lambda_{i}\left[f_{i}\left(u^{1}, v^{1}\right)+g_{i}\left(u^{2}, v^{2}\right)-s\left(v^{1} \mid D_{i}^{1}\right)-s\left(v^{2} \mid D_{i}^{2}\right)+\left(u^{1}\right)^{T} w_{i}^{1}\right. \\
& \left.+\left(u^{2}\right)^{T} w_{i}^{2}-\frac{1}{2}\left(q_{i}^{1}\right)^{T} \nabla_{u^{1} u^{1}} f_{i}\left(u^{1}, v^{1}\right) q_{i}^{1}-\frac{1}{2}\left(q_{i}^{2}\right)^{T} \nabla_{u^{2} u^{2}} g_{i}\left(u^{2}, v^{2}\right) q_{i}^{2}\right]
\end{aligned}
$$

By the second order $F_{1}$-convexity of $f_{i}\left(\cdot, v^{1}\right)+\cdot{ }^{T} w_{i}^{1}$ at $x^{1}$ with respect to $q_{i}^{1} \in \mathbb{R}^{\left|J_{1}\right|}$, we have

$$
\begin{aligned}
& f_{i}\left(x^{1}, v^{1}\right)+\left(x^{1}\right)^{T} w_{i}^{1}-f_{i}\left(u^{1}, v^{1}\right)-\left(u^{1}\right)^{T} w_{i}^{1}+\frac{1}{2}\left(q_{i}^{1}\right)^{T} \nabla_{u^{1} u^{1}} f_{i}\left(u^{1}, v^{1}\right) q_{i}^{1} \\
& \geqq F_{1}\left(x^{1}, u^{1} ; \nabla_{u^{1}} f_{i}\left(u^{1}, v^{1}\right)+w_{i}^{1}+\nabla_{u^{1} u^{1}} f_{i}\left(u^{1}, v^{1}\right) q_{i}^{1}\right) .
\end{aligned}
$$


for $i=1,2, \ldots, l$.

From $\lambda>0$ and sublinearity of $F_{1}$, we have

$$
\begin{aligned}
& \sum_{i=1}^{l} \lambda_{i}\left[f_{i}\left(x^{1}, v^{1}\right)+\left(x^{1}\right)^{T} w_{i}^{1}-f_{i}\left(u^{1}, v^{1}\right)-\left(u^{1}\right)^{T} w_{i}^{1}\right. \\
& \left.+\frac{1}{2}\left(q_{i}^{1}\right)^{T} \nabla_{u^{1} u^{1}} f_{i}\left(u^{1}, v^{1}\right) q_{i}^{1}\right] \\
& \geqq F_{1}\left(x^{1}, u^{1} ; \sum_{i=1}^{l} \lambda_{i}\left[\nabla_{u^{1}} f_{i}\left(u^{1}, v^{1}\right)+w_{i}^{1}+\nabla_{u^{1} u^{1}} f_{i}\left(u^{1}, v^{1}\right) q_{i}^{1}\right]\right) .
\end{aligned}
$$

By the duality constraint (8), it follows that

$$
a=\sum_{i=1}^{l} \lambda_{i}\left[\nabla_{u^{1}} f_{i}\left(u^{1}, v^{1}\right)+w_{i}^{1}+\nabla_{u^{1} u^{1}} f_{i}\left(u^{1}, v^{1}\right) q_{i}^{1}\right] \in \mathbb{R}_{+}^{\left|J_{1}\right|} .
$$

Thus, by condition (i) given in Theorem 1, we have

$$
F_{1}\left(x^{1}, u^{1} ; a\right)+\left(u^{1}\right)^{T} a \geqq 0,
$$

that is,

$$
\begin{aligned}
& F_{1}\left(x^{1}, u^{1} ; \sum_{i=1}^{l} \lambda_{i}\left[\nabla_{u^{1}} f_{i}\left(u^{1}, v^{1}\right)+w_{i}^{1}+\nabla_{u^{1} u^{1}} f_{i}\left(u^{1}, v^{1}\right) q_{i}^{1}\right]\right) \\
& \geqq-\left(u^{1}\right)^{T} \sum_{i=1}^{l} \lambda_{i}\left[\nabla_{u^{1}} f_{i}\left(u^{1}, v^{1}\right)+w_{i}^{1}+\nabla_{u^{1} u^{1}} f_{i}\left(u^{1}, v^{1}\right) q_{i}^{1}\right] .
\end{aligned}
$$

From (10), (16) and (17), we get

$$
\begin{aligned}
& \sum_{i=1}^{l} \lambda_{i}\left[f_{i}\left(x^{1}, v^{1}\right)+\left(x^{1}\right)^{T} w_{i}^{1}-f_{i}\left(u^{1}, v^{1}\right)-\left(u^{1}\right)^{T} w_{i}^{1}\right. \\
& \left.+\frac{1}{2}\left(q_{i}^{1}\right)^{T} \nabla_{u^{1} u^{1}} f_{i}\left(u^{1}, v^{1}\right) q_{i}^{1}\right] \geqq 0 .
\end{aligned}
$$

By second order $F_{2}$-concavity of $f_{i}\left(x^{1}, \cdot\right)-{ }^{T} z_{i}^{1}$ for fixed $x^{1}$, with respect to $p_{i}^{1} \in \mathbb{R}^{\left|K_{1}\right|}$, we have

$$
\begin{aligned}
& -f_{i}\left(x^{1}, v^{1}\right)+\left(v^{1}\right)^{T} z_{i}^{1}+f_{i}\left(x^{1}, y^{1}\right)-\left(y^{1}\right)^{T} z_{i}^{1}-\frac{1}{2}\left(p_{i}^{1}\right)^{T} \nabla_{y^{1} y^{1}} f_{i}\left(x^{1}, y^{1}\right) p_{i}^{1} \\
& \geqq F_{2}\left(v^{1}, y^{1} ;-\nabla_{y^{1}} f_{i}\left(x^{1}, y^{1}\right)+z_{i}^{1}-\nabla_{y^{1} y^{1}} f_{i}\left(x^{1}, y^{1}\right) p_{i}^{1}\right) .
\end{aligned}
$$

From $\lambda>0$ and sublinearity of $F_{2}$, we have

$$
\sum_{i=1}^{l} \lambda_{i}\left[f_{i}\left(x^{1}, y^{1}\right)-\left(y^{1}\right)^{T} z_{i}^{1}+\left(v^{1}\right)^{T} z_{i}^{1}-f_{i}\left(x^{1}, v^{1}\right)\right.
$$




$$
\begin{aligned}
& \left.-\frac{1}{2}\left(p_{i}^{1}\right)^{T} \nabla_{y^{1} y^{1}} f_{i}\left(x^{1}, y^{1}\right) p_{i}^{1}\right] \\
\geqq & F_{2}\left(v^{1}, y^{1} ; \sum_{i=1}^{l} \lambda_{i}\left[-\nabla_{y^{1}} f_{i}\left(x^{1}, y^{1}\right)+z_{i}^{1}-\nabla_{y^{1} y^{1}} f_{i}\left(x^{1}, y^{1}\right) p_{i}^{1}\right]\right) .
\end{aligned}
$$

By the primal constraint (1), it follows that

$$
c=-\sum_{i=1}^{l} \lambda_{i}\left[\nabla_{y^{1}} f_{i}\left(x^{1}, y^{1}\right)-z_{i}^{1}+\nabla_{y^{1} y^{1}} f_{i}\left(x^{1}, y^{1}\right) p_{i}^{1}\right] \geqq 0 .
$$

Thus, by condition (iii) given in Theorem 1, we have

$$
F_{2}\left(v^{1}, y^{1} ; c\right)+\left(y^{1}\right)^{T} c \geqq 0,
$$

that is,

$$
\begin{aligned}
& F_{2}\left(v^{1}, y^{1} ;-\sum_{i=1}^{l} \lambda_{i}\left[\nabla_{y^{1}} f_{i}\left(x^{1}, y^{1}\right)-z_{i}^{1}+\nabla_{y^{1} y^{1}} f_{i}\left(x^{1}, y^{1}\right) p_{i}^{1}\right]\right) \\
& \geqq\left(y^{1}\right)^{T} \sum_{i=1}^{l} \lambda_{i}\left[\nabla_{y^{1}} f_{i}\left(x^{1}, y^{1}\right)-z_{i}^{1}+\nabla_{y^{1} y^{1}} f_{i}\left(x^{1}, y^{1}\right) p_{i}^{1}\right] .
\end{aligned}
$$

From (3), (19) and (20), we get

$$
\begin{aligned}
& \sum_{i=1}^{l} \lambda_{i}\left[f_{i}\left(x^{1}, y^{1}\right)-\left(y^{1}\right)^{T} z_{i}^{1}+\left(v^{1}\right)^{T} z_{i}^{1}-f_{i}\left(x^{1}, v^{1}\right)\right. \\
& \left.-\frac{1}{2}\left(p_{i}^{1}\right)^{T} \nabla_{y^{1} y^{1}} f_{i}\left(x^{1}, y^{1}\right) p_{i}^{1}\right] \geqq 0 .
\end{aligned}
$$

Using $\left(x^{1}\right)^{T} w_{i}^{1} \leqq s\left(x^{1} \mid C_{i}^{1}\right)$ and $\left(v^{1}\right)^{T} z_{i}^{1} \leqq s\left(v^{1} \mid D_{i}^{1}\right)$, it follows from (18) and $(21)$, that

$$
\begin{aligned}
& \sum_{i=1}^{l} \lambda_{i}\left[f_{i}\left(x^{1}, y^{1}\right)+s\left(x^{1} \mid C_{i}^{1}\right)-\left(y^{1}\right)^{T} z_{i}^{1}\right. \\
& \left.-\frac{1}{2}\left(p_{i}^{1}\right)^{T} \nabla_{y^{1} y^{1}} f_{i}\left(x^{1}, y^{1}\right) p_{i}^{1}\right] \\
& \geqq \sum_{i=1}^{l} \lambda_{i}\left[f_{i}\left(u^{1}, v^{1}\right)-s\left(v^{1} \mid D_{i}^{1}\right)+\left(u^{1}\right)^{T} w_{i}^{1}-\frac{1}{2}\left(q_{i}^{1}\right)^{T} \nabla_{u^{1} u^{1}} f_{i}\left(u^{1}, v^{1}\right) q_{i}^{1}\right]
\end{aligned}
$$

Similarly, using remaining hypotheses and conditions given in Theorem 1 and using constraints of the primal and dual problems for functions 
$g_{i}\left(\cdot, v^{2}\right)+{ }^{T} w_{i}^{2}$ and $g_{i}\left(x^{2}, \cdot\right)-{ }^{T} z_{i}^{2}$, we get

$$
\begin{aligned}
& \sum_{i=1}^{l} \lambda_{i}\left[g_{i}\left(x^{2}, y^{2}\right)+s\left(x^{2} \mid C_{i}^{2}\right)-\left(y^{2}\right)^{T} z_{i}^{2}\right. \\
& \left.-\frac{1}{2}\left(p_{i}^{2}\right)^{T} \nabla_{y^{2} y^{2}} g_{i}\left(x^{2}, y^{2}\right) p_{i}^{2}\right] \\
& \geqq \sum_{i=1}^{l} \lambda_{i}\left[g_{i}\left(u^{2}, v^{2}\right)-s\left(v^{2} \mid D_{i}^{2}\right)+\left(u^{2}\right)^{T} w_{i}^{2}-\frac{1}{2}\left(q_{i}^{2}\right)^{T} \nabla_{u^{2} u^{2}} f_{i}\left(u^{2}, v^{2}\right) q_{i}^{2}\right] .
\end{aligned}
$$

From (22) and (23), we get

$$
\begin{aligned}
& \sum_{i=1}^{l} \lambda_{i}\left[f_{i}\left(x^{1}, y^{1}\right)+g_{i}\left(x^{2}, y^{2}\right)+s\left(x^{1} \mid C_{i}^{1}\right)+s\left(x^{2} \mid C_{i}^{2}\right)-\left(y^{1}\right)^{T} z_{i}^{1}\right. \\
& \left.-\left(y^{2}\right)^{T} z_{i}^{2}-\frac{1}{2}\left(p_{i}^{1}\right)^{T} \nabla_{y^{1} y^{1}} f_{i}\left(x^{1}, y^{1}\right) p_{i}^{1}-\frac{1}{2}\left(p_{i}^{2}\right)^{T} \nabla_{y^{2} y^{2}} g_{i}\left(x^{2}, y^{2}\right) p_{i}^{2}\right] \\
& \geqq \sum_{i=1}^{l} \lambda_{i}\left[f_{i}\left(u^{1}, v^{1}\right)+g_{i}\left(u^{2}, v^{2}\right)-s\left(v^{1} \mid D_{i}^{1}\right)-s\left(v^{2} \mid D_{i}^{2}\right)+\left(u^{1}\right)^{T} w_{i}^{1}\right. \\
& \left.+\left(u^{2}\right)^{T} w_{i}^{2}-\frac{1}{2}\left(q_{i}^{1}\right)^{T} \nabla_{u^{1} u^{1}} f_{i}\left(u^{1}, v^{1}\right) q_{i}^{1}-\frac{1}{2}\left(q_{i}^{2}\right)^{T} \nabla_{u^{2} u^{2}} g_{i}\left(u^{2}, v^{2}\right) q_{i}^{2}\right],
\end{aligned}
$$

which is a contradiction to (15).

Hence

$$
H\left(x^{1}, x^{2}, y^{1}, y^{2}, z^{1}, z^{2}, p^{1}, p^{2}, \lambda\right) \not \leq G\left(u^{1}, u^{2}, v^{1}, v^{2}, w^{1}, w^{2}, q^{1}, q^{2}, \lambda\right) .
$$

Theorem 2 (Weak duality). Let $\left(x^{1}, x^{2}, y^{1}, y^{2}, z^{1}, z^{2}, p^{1}, p^{2}, \lambda\right)$ be feasible for $(S M P)$ and $\left(u^{1}, u^{2}, v^{1}, v^{2}, w^{1}, w^{2}, q^{1}, q^{2}, \lambda\right)$ be feasible for $(S M D)$. Suppose there exist sublinear functionals $F_{1}, F_{2}, G_{1}$ and $G_{2}$ satisfying the following conditions:

(i) $F_{1}\left(x^{1}, u^{1} ; a\right)+\left(u^{1}\right)^{T} a \geqq 0$ if $a \geqq 0$;

(ii) $G_{1}\left(x^{2}, u^{2} ; b\right)+\left(u^{2}\right)^{T} b \geqq 0$ if $b \geqq 0$;

(iii) $F_{2}\left(v^{1}, y^{1} ; c\right)+\left(y^{1}\right)^{T} c \geqq 0$ if $c \geqq 0$;

(iv) $G_{2}\left(v^{2}, y^{2} ; d\right)+\left(y^{2}\right)^{T} d \geqq 0$ if $d \geqq 0$.

Furthermore, assume that for $i=1,2, \ldots, l, f_{i}\left(\cdot, v^{1}\right)+{ }^{T} w_{i}^{1}$ is second order $F_{1}$-pseudo-convex for fixed $v^{1}$, with respect to $q_{i}^{1} \in \mathbb{R}^{\left|J_{1}\right|}, f_{i}\left(x^{1}, \cdot\right)-{ }^{T} z_{i}^{1}$ is second order $F_{2}$-pseudo-concave for fixed $x^{1}$, with respect to $p_{i}^{1} \in \mathbb{R}^{\left|K_{1}\right|}$, $g_{i}\left(\cdot, v^{2}\right)+{ }^{T} w_{i}^{2}$ is second order $G_{1}$-pseudo-convex for fixed $v^{2}$ with respect 
to $q_{i}^{2} \in \mathbb{R}^{\left|J_{2}\right|}$ and $g_{i}\left(x^{2}, \cdot\right)-\cdot^{T} z_{i}^{2}$ is second order $G_{2}$-pseudo-concave for fixed $x^{2}$, with respect to $p_{i}^{2} \in \mathbb{R}^{\left|K_{2}\right|}$.

Then

$$
H\left(x^{1}, x^{2}, y^{1}, y^{2}, z^{1}, z^{2}, p^{1}, p^{2}, \lambda\right) \not \leq G\left(u^{1}, u^{2}, v^{1}, v^{2}, w^{1}, w^{2}, q^{1}, q^{2}, \lambda\right) .
$$

Proof. Assume that the result is not true, that is,

$$
H\left(x^{1}, x^{2}, y^{1}, y^{2}, z^{1}, z^{2}, p^{1}, p^{2}, \lambda\right) \leq G\left(u^{1}, u^{2}, v^{1}, v^{2}, w^{1}, w^{2}, q^{1}, q^{2}, \lambda\right) .
$$

Then, since $\lambda>0$, we have

$$
\begin{aligned}
& \sum_{i=1}^{l} \lambda_{i}\left[f_{i}\left(x^{1}, y^{1}\right)+g_{i}\left(x^{2}, y^{2}\right)+s\left(x^{1} \mid C_{i}^{1}\right)+s\left(x^{2} \mid C_{i}^{2}\right)-\left(y^{1}\right)^{T} z_{i}^{1}\right. \\
& -\left(y^{2}\right)^{T} z_{i}^{2}-\frac{1}{2}\left(p_{i}^{1}\right)^{T} \nabla_{y^{1} y^{1}} f_{i}\left(x^{1}, y^{1}\right) p_{i}^{1} \\
& \left.-\frac{1}{2}\left(p_{i}^{2}\right)^{T} \nabla_{y^{2} y^{2}} g_{i}\left(x^{2}, y^{2}\right) p_{i}^{2}\right] \\
& <\sum_{i=1}^{l} \lambda_{i}\left[f_{i}\left(u^{1}, v^{1}\right)+g_{i}\left(u^{2}, v^{2}\right)-s\left(v^{1} \mid D_{i}^{1}\right)-s\left(v^{2} \mid D_{i}^{2}\right)+\left(u^{1}\right)^{T} w_{i}^{1}\right. \\
& \left.+\left(u^{2}\right)^{T} w_{i}^{2}-\frac{1}{2}\left(q_{i}^{1}\right)^{T} \nabla_{u^{1} u^{1}} f_{i}\left(u^{1}, v^{1}\right) q_{i}^{1}-\frac{1}{2}\left(q_{i}^{2}\right)^{T} \nabla_{u^{2} u^{2}} g_{i}\left(u^{2}, v^{2}\right) q_{i}^{2}\right]
\end{aligned}
$$

By the duality constraint (8), it follows that

$$
a=\sum_{i=1}^{l} \lambda_{i}\left[\nabla_{u^{1}} f_{i}\left(u^{1}, v^{1}\right)+w_{i}^{1}+\nabla_{u^{1} u^{1}} f_{i}\left(u^{1}, v^{1}\right) q_{i}^{1}\right] \in \mathbb{R}_{+}^{\left|J_{1}\right|} .
$$

Thus, by condition (i) given in Theorem 2, we have

$$
F_{1}\left(x^{1}, u^{1} ; a\right)+\left(u^{1}\right)^{T} a \geqq 0,
$$

that is,

$$
\begin{aligned}
& F_{1}\left(x^{1}, u^{1} ; \sum_{i=1}^{l} \lambda_{i}\left[\nabla_{u^{1}} f_{i}\left(u^{1}, v^{1}\right)+w_{i}^{1}+\nabla_{u^{1} u^{1}} f_{i}\left(u^{1}, v^{1}\right) q_{i}^{1}\right]\right) \\
& \geqq-\left(u^{1}\right)^{T} \sum_{i=1}^{l} \lambda_{i}\left[\nabla_{u^{1}} f_{i}\left(u^{1}, v^{1}\right)+w_{i}^{1}+\nabla_{u^{1} u^{1}} f_{i}\left(u^{1}, v^{1}\right) q_{i}^{1}\right]
\end{aligned}
$$

From (10) and (25), we get

$$
F_{1}\left(x^{1}, u^{1} ; \sum_{i=1}^{l} \lambda_{i}\left[\nabla_{u^{1}} f_{i}\left(u^{1}, v^{1}\right)+w_{i}^{1}+\nabla_{u^{1} u^{1}} f_{i}\left(u^{1}, v^{1}\right) q_{i}^{1}\right]\right) \geqq 0 .
$$


From (26) and second order $F_{1}$-pseudo-convexity of $f_{i}\left(\cdot, v^{1}\right)+\cdot{ }^{T} w_{i}^{1}$ for fixed $v^{1}$, with respect to $q_{i}^{1} \in \mathbb{R}^{\left|J_{1}\right|}$, we get

$$
\begin{aligned}
& \sum_{i=1}^{l} \lambda_{i}\left[f_{i}\left(x^{1}, v^{1}\right)+\left(x^{1}\right)^{T} w_{i}^{1}-f_{i}\left(u^{1}, v^{1}\right)-\left(u^{1}\right)^{T} w_{i}^{1}\right. \\
& \left.+\frac{1}{2}\left(q_{i}^{1}\right)^{T} \nabla_{u^{1} u^{1}} f_{i}\left(u^{1}, v^{1}\right) q_{i}^{1}\right] \geqq 0 .
\end{aligned}
$$

By primal constraint (1), it follows that

$$
c=-\sum_{i=1}^{l} \lambda_{i}\left[\nabla_{y^{1}} f_{i}\left(x^{1}, y^{1}\right)-z_{i}^{1}+\nabla_{y^{1} y^{1}} f_{i}\left(x^{1}, y^{1}\right) p_{i}^{1}\right] \geqq 0 .
$$

Thus, by condition (iii) given in Theorem 2, we have

$$
F_{2}\left(v^{1}, y^{1} ; c\right)+\left(y^{1}\right)^{T} c \geqq 0
$$

that is,

$$
\begin{aligned}
& F_{2}\left(v^{1}, y^{1} ;-\sum_{i=1}^{l} \lambda_{i}\left[\nabla_{y^{1}} f_{i}\left(x^{1}, y^{1}\right)-z_{i}^{1}+\nabla_{y^{1} y^{1}} f_{i}\left(x^{1}, y^{1}\right) p_{i}^{1}\right]\right) \\
& \geqq\left(y^{1}\right)^{T} \sum_{i=1}^{l} \lambda_{i}\left[\nabla_{y^{1}} f_{i}\left(x^{1}, y^{1}\right)-z_{i}^{1}+\nabla_{y^{1} y^{1}} f_{i}\left(x^{1}, y^{1}\right) p_{i}^{1}\right] .
\end{aligned}
$$

From (3) and (28), we get

$$
F_{2}\left(v^{1}, y^{1} ;-\sum_{i=1}^{l} \lambda_{i}\left[\nabla_{y^{1}} f_{i}\left(x^{1}, y^{1}\right)-z_{i}^{1}+\nabla_{y^{1} y^{1}} f_{i}\left(x^{1}, y^{1}\right) p_{i}^{1}\right]\right) \geqq 0 .
$$

From (29) and the second order $F_{2}$-pseudo-concavity of $f_{i}\left(x^{1}, \cdot\right)-\cdot^{T} z_{i}^{1}$ for fixed $x^{1}$, with respect to $p_{i}^{1} \in \mathbb{R}^{\left|K_{1}\right|}$, we have

$$
\begin{aligned}
& \sum_{i=1}^{l} \lambda_{i}\left[f_{i}\left(x^{1}, y^{1}\right)-\left(y^{1}\right)^{T} z_{i}^{1}+\left(v^{1}\right)^{T} z_{i}^{1}-f_{i}\left(x^{1}, v^{1}\right)\right. \\
& \left.-\frac{1}{2}\left(p_{i}^{1}\right)^{T} \nabla_{y^{1} y^{1}} f_{i}\left(x^{1}, y^{1}\right) p_{i}^{1}\right] \geqq 0 .
\end{aligned}
$$

Using $\left(x^{1}\right)^{T} w_{i}^{1} \leqq s\left(x^{1} \mid C_{i}^{1}\right)$ and $\left(v^{1}\right)^{T} z_{i}^{1} \leqq s\left(v^{1} \mid D_{i}^{1}\right)$, it follows from (27) and (30), that

$$
\begin{aligned}
& \sum_{i=1}^{l} \lambda_{i}\left[f_{i}\left(x^{1}, y^{1}\right)+s\left(x^{1} \mid C_{i}^{1}\right)-\left(y^{1}\right)^{T} z_{i}^{1}\right. \\
& \left.-\frac{1}{2}\left(p_{i}^{1}\right)^{T} \nabla_{y^{1} y^{1}} f_{i}\left(x^{1}, y^{1}\right) p_{i}^{1}\right]
\end{aligned}
$$




$$
\geqq \sum_{i=1}^{l} \lambda_{i}\left[f_{i}\left(u^{1}, v^{1}\right)-s\left(v^{1} \mid D_{i}^{1}\right)+\left(u^{1}\right)^{T} w_{i}^{1}-\frac{1}{2}\left(q_{i}^{1}\right)^{T} \nabla_{u^{1} u^{1}} f_{i}\left(u^{1}, v^{1}\right) q_{i}^{1}\right] .
$$

Similarly, using remaining hypotheses and conditions given in Theorem 2 and using constraints of the primal and dual problems for functions $g_{i}\left(\cdot, v^{2}\right)+{ }^{T} w_{i}^{2}$ and $g_{i}\left(x^{2}, \cdot\right)-\cdot{ }^{T} z_{i}^{2}$, we get

$$
\begin{aligned}
& \sum_{i=1}^{l} \lambda_{i}\left[g_{i}\left(x^{2}, y^{2}\right)+s\left(x^{2} \mid C_{i}^{2}\right)-\left(y^{2}\right)^{T} z_{i}^{2}\right. \\
& \left.-\frac{1}{2}\left(p_{i}^{2}\right)^{T} \nabla_{y^{2} y^{2}} g_{i}\left(x^{2}, y^{2}\right) p_{i}^{2}\right] \\
& \geqq \sum_{i=1}^{l} \lambda_{i}\left[g_{i}\left(u^{2}, v^{2}\right)-s\left(v^{2} \mid D_{i}^{2}\right)+\left(u^{2}\right)^{T} w_{i}^{2}-\frac{1}{2}\left(q_{i}^{2}\right)^{T} \nabla_{u^{2} u^{2}} f_{i}\left(u^{2}, v^{2}\right) q_{i}^{2}\right] .
\end{aligned}
$$

From (31) and (32), we get

$$
\begin{aligned}
& \sum_{i=1}^{l} \lambda_{i}\left[f_{i}\left(x^{1}, y^{1}\right)+g_{i}\left(x^{2}, y^{2}\right)+s\left(x^{1} \mid C_{i}^{1}\right)+s\left(x^{2} \mid C_{i}^{2}\right)-\left(y^{1}\right)^{T} z_{i}^{1}\right. \\
& \left.-\left(y^{2}\right)^{T} z_{i}^{2}-\frac{1}{2}\left(p_{i}^{1}\right)^{T} \nabla_{y^{1} y^{1}} f_{i}\left(x^{1}, y^{1}\right) p_{i}^{1}-\frac{1}{2}\left(p_{i}^{2}\right)^{T} \nabla_{y^{2} y^{2}} g_{i}\left(x^{2}, y^{2}\right) p_{i}^{2}\right] \\
& \geqq \sum_{i=1}^{l} \lambda_{i}\left[f_{i}\left(u^{1}, v^{1}\right)+g_{i}\left(u^{2}, v^{2}\right)-s\left(v^{1} \mid D_{i}^{1}\right)-s\left(v^{2} \mid D_{i}^{2}\right)+\left(u^{1}\right)^{T} w_{i}^{1}\right. \\
& \left.+\left(u^{2}\right)^{T} w_{i}^{2}-\frac{1}{2}\left(q_{i}^{1}\right)^{T} \nabla_{u^{1} u^{1}} f_{i}\left(u^{1}, v^{1}\right) q_{i}^{1}-\frac{1}{2}\left(q_{i}^{2}\right)^{T} \nabla_{u^{2} u^{2}} g_{i}\left(u^{2}, v^{2}\right) q_{i}^{2}\right]
\end{aligned}
$$

which is a contradiction to (24).

Hence

$$
H\left(x^{1}, x^{2}, y^{1}, y^{2}, z^{1}, z^{2}, p^{1}, p^{2}, \lambda\right) \not \leq G\left(u^{1}, u^{2}, v^{1}, v^{2}, w^{1}, w^{2}, q^{1}, q^{2}, \lambda\right) .
$$

Theorem 3 (Strong duality). Let $\left(\overline{x^{1}}, \overline{x^{2}}, \overline{y^{1}}, \overline{y^{2}}, \overline{z^{1}}, \overline{z^{2}}, \overline{p^{1}}, \overline{p^{2}}, \bar{\lambda}\right)$ be $a$ properly efficient solution for $(S M P)$. Let $\bar{\lambda}=\lambda$ be fixed in $(S M D)$. Suppose that the Hessian matrix $\nabla_{y^{1}}^{2} f_{i}\left(\overline{x^{1}}, \overline{y^{1}}\right)$ is positive definite for $i=1,2, \ldots, l$ and $\sum_{i=1}^{l} \lambda_{i}\left(p_{i}^{1}\right)^{T}\left[\nabla_{y^{1}} g_{i}-\bar{z}_{i}^{1}\right] \geqq 0$; and $\nabla_{y^{2}}^{2} g_{i}\left(\overline{x^{2}}, \overline{y^{2}}\right)$ is positive definite for $i=1,2, \ldots, l$ and $\sum_{i=1}^{l} \lambda_{i}\left(p_{i}^{2}\right)^{T}\left[\nabla_{y^{2}} g_{i}-\bar{z}_{i}^{2}\right] \geqq 0 ;$ or $\nabla_{y^{1}}^{2} f_{i}\left(\overline{x^{1}}, \overline{y^{1}}\right)$ is negative definite for $i=1,2, \ldots, l$ and $\sum_{i=1}^{l} \lambda_{i}\left(p_{i}^{1}\right)^{T}\left[\nabla_{y^{1}} f_{i}-\bar{z}_{i}^{1}\right] \leqq 0$; 
and $\nabla_{y^{2}}^{2} g_{i}\left(\overline{x^{2}}, \overline{y^{2}}\right)$ is negative definite for $i=1,2, \ldots, l$ and $\sum_{i=1}^{l} \lambda_{i}\left(p_{i}^{2}\right)^{T}\left[\nabla_{y^{2}} g_{i}-\bar{z}_{i}^{2}\right] \leqq 0$. Also suppose that the sets

$$
\left\{\nabla_{y^{1}} f_{1}-\bar{z}_{1}^{2}+\nabla_{y^{1} y^{1}} f_{1} \bar{p}_{1}^{1}, \ldots, \nabla_{y^{1}} f_{l}-\bar{z}_{l}^{2}+\nabla_{y^{1} y^{1}} f_{l} \bar{p}_{l}^{1}\right\}
$$

and

$$
\left\{\nabla_{y^{2}} g_{1}-\bar{z}_{1}^{2}+\nabla_{y^{2} y^{2}} g_{1} \bar{p}_{1}^{2}, \ldots, \nabla_{y^{2}} g_{l}-\bar{z}_{l}^{2}+\nabla_{y^{2} y^{2}} g_{l} \bar{p}_{l}^{2}\right\}
$$

are linearly independent. Then there exists $w_{i}^{1} \in C_{i}^{1}$ and $w_{i}^{2} \in C_{i}^{2}$ such that

$$
\left(\overline{x^{1}}, \overline{x^{2}}, \overline{y^{1}}, \overline{y^{2}}, \overline{w^{1}}, \overline{w^{2}}, \overline{q^{1}}=\overline{q^{2}}=0, \bar{\lambda}\right)
$$

is feasible for $(S M D)$ and

$$
H\left(x^{1}, x^{2}, y^{1}, y^{2}, z^{1}, z^{2}, p^{1}, p^{2}, \lambda\right)=G\left(u^{1}, u^{2}, v^{1}, v^{2}, w^{1}, w^{2}, q^{1}, q^{2}, \lambda\right) .
$$

Moreover, if the generalized convexity hypotheses and conditions (i)-(iv) of Theorem 1 or 2 are satisfied, then $\left(\overline{x^{1}}, \overline{x^{2}}, \overline{y^{1}}, \overline{y^{2}}, \overline{w^{1}}, \overline{w^{2}}, \overline{q^{1}}, \overline{q^{2}}, \bar{\lambda}\right)$ is a properly efficient solution for $(S M D)$.

Proof. The proof follows the lines of the proof of Theorem 2 in Yang et al. [28] in light of the discussions above in this paper.

Remark 2. The converse duality theorem can also be established for the problems considered in this paper.

\section{Special Cases}

In this section, we consider some special cases of the problem (SMP) and (SMD) by choosing particular forms of the sublinear functionals and the compact convex sets involved in the problems.

If $\left|J_{2}\right|=\left|K_{2}\right|=0$, then (SMP) and (SMD) reduce to the problems (P) and (D) studied by Yang et al. [28].

If $\left|J_{2}\right|=\left|K_{2}\right|=0$, and $C_{i}^{1}=C_{i}^{2}=D_{i}^{1}=D_{i}^{2}=\{0\}, i=1,2, \ldots, l$, then $(\mathrm{SMP})$ and $(\mathrm{SMD})$ reduce to the problems $(\mathrm{P})$ and $(\mathrm{D})$ studied by Suneja et al. [24].

If $C_{i}^{1}=C_{i}^{2}=D_{i}^{1}=D_{i}^{2}=\{0\}, i=1,2, \ldots, l$, and $l=1$, then (SMP) and $(\mathrm{SMD})$ reduce to the problems (MP) and (MD) studied by Mishra [15].

If $\left|J_{2}\right|=\left|K_{2}\right|=0$, and $l=1$, then (SMP) and (SMD) reduce to the pair of problems studied by Hou and Yang [11].

If $\left|J_{2}\right|=\left|K_{2}\right|=0, l=1$, and $p^{1}=p^{2}=q^{1}=q^{2}=0$ then (SMP) and (SMD) reduce to the problems (P1) and (D1) studied by Mond and Schechter [20]. 
If $l=1, p^{1}=p^{2}=q^{1}=q^{2}=0$ and $\nabla_{2} f\left(x^{1}, y^{1}\right)=z^{1}$, then (SMP) and $(\mathrm{SMD})$ reduce to the problems $(\mathrm{MP})$ and $(\mathrm{MD})$ studied by Yang et al. [27].

If $p_{i}^{1}=p_{i}^{2}=q_{i}^{1}=q_{i}^{2}=0$ and $C_{i}^{1}=C_{i}^{2}=D_{i}^{1}=D_{i}^{2}=\{0\}, i=1,2, \ldots, l$, and then (SMP) and (SMD) reduce to a general form of the problems (P1) and (D1) studied by Bector et al. [3].

\section{Conclusion}

In this paper, we have extended the results of Yang et al. [28], Suneja et al. [24], Hou and Yang [11] and Mond and Schechter [20] to mixed symmetric second order duality, the results of Bector et al. [3] to the case of non-differentiable problems and the results of Yang et al. [27] to the second order case. The results obtained in this paper can be extended to the class of functions introduced by Antczak [2] and Aghezzaf and Hachimi [1]. Moreover, these results can be further extended to higher order case as an extension of the results of Chen [4], to the case of complex functions and to the case of continuous-time problems as well. Some of these problems will be the subject of research of the present author in very near future.

Acknowledgement. The author is grateful to the two anonymous referees for their constructive suggestion and very careful reading of the previous version of the manuscript.

\section{References}

[1] Aghezzaf, B., Hachimi, M., Sufficient optimality conditions and duality in multiobjective programming involving generalized convexity, Numer. Funct. Anal. Optim. 22 (2001), 775-788.

[2] Antczak, T., Multiobjective programming under d-invexity, European J. Oper. Res. 137 (2002), 28-36.

[3] Bector, C. R., Chandra, S., Abha, On mixed symmetric duality in multiobjective programming, Opsearch 36 (1999), 399-407.

[4] Chen, X., Higher-order symmetric duality in nondifferentiable multiobjective programming problems, J. Math. Anal. Appl. 290 (2004), 423-435.

[5] Dantzig, G. B., Eisenberg, E., Cottle, R. W., Symmetric dual nonlinear programs, Pacific J. Math. 15 (1965), 809-812.

[6] Devi, G., Symmetric duality for nonlinear programming problem involving $\eta$-convex functions, European J. Oper. Res. 104 (1998), 615-621.

[7] Dorn, W. S., A symmetric dual theorem for quadratic programming, J. Oper. Res. Soc. Japan 2 (1960). 93-97.

[8] Hanson, M. A., On sufficiency of the Kuhn-Tucker conditions, J. Math. Anal. Appl. 80 (1981), 545-550.

[9] Hanson, M. A., Second order invexity and duality in mathematical programming, Opsearch 30 (1993), 313-320. 
[10] Hanson, M. A., Mond, B., Further generalization of convexity in mathematical programming, J. Inf. Optim. Sci. 3 (1982), 25-32.

[11] Hou, S. H., Yang, X. M., On second order symmetric duality in nondifferentiable programming, J. Math. Anal. Appl. 255 (2001), 491-498.

[12] Kaul, R. N., Kaur, S., Optimality criteria in nonlinear programming involving nonconvex functions, J. Math. Anal. Appl. 105 (1985), 104-112.

[13] Mangasarian, O. L., Second and higher order duality in nonlinear programming, J. Math. Anal. Appl. 51 (1975), 607-620.

[14] Mishra, S. K., Multiobjective second order symmetric duality with cone constraints, European J. Oper. Res. 126 (2000), 675-682.

[15] Mishra, S. K., Second order symmetric duality in mathematical programming with F-convexity, European J. Oper. Res. 127 (2000), 507-518.

[16] Mishra, S. K., On second order symmetric duality in mathematical programming, in: "Recent Developments in Operational Research", M. L. Agarwal and K. Sen (Eds.), Narosa Publishing House, New Delhi, 2001, 261-272.

[17] Mishra, S. K., Second order generalized invexity and duality in mathematical programming, Optimization 42 (1997), 51-69.

[18] Mond, B., A symmetric dual theorem for nonlinear programs, Quart. Appl. Math. 23 (1965), 265-269.

[19] Mond, B., Second order duality for nonlinear programs, Opsearch 11 (1974), 90-99.

[20] Mond, B., Schechter, M., Nondifferentiable symmetric duality, Bull. Austral. Math. Soc. 53 (1996), 177-188.

[21] Mond, B., Weir, T., Symmetric duality for nonlinear multiobjective programming, in: "Recent Developments in Mathematical Programming", S. Kumar (Ed.), Gordon and Breach, London (1991), 137-153.

[22] Nanda, S., Das, L. N., Pseudo-invexity and duality in nonlinear programming, European J. Oper. Res. 88 (1996), 572-577.

[23] Rockafellar, R. T., Convex Analysis, Princeton University Press, Princeton, NJ, 1970.

[24] Suneja, S. K., Lalitha, C. S., Khurana, S., Second order symmetric duality in multiobjective programming, European J. Oper. Res. 144 (2003), 492-500.

[25] Weir, T., Mond, B., Symmetric and self duality in multiobjective programming, AsiaPacific J. Oper. Res. 5 (1988), 124-133.

[26] Yang, X. M., Yang, X. Q., Teo, K. L., Nondifferentiable second order symmetric duality in mathematical programming with F-convexity, European J. Oper. Res. 144 (2003), 554-559.

[27] Yang, X. M., Teo, K. L., Yang, X. Q., Mixed symmetric duality in nondifferentiable mathematical programming, Indian J. Pure Appl. Math. 34 (2003), 805-815.

[28] Yang, X. M., Yang, X. Q., Teo, K. L., Hou, S. H., Second order symmetric duality in non-differentiable multi-objective programming with F-convexity, European J. Oper. Res. 164 (2005), 406-416.

S. K. Mishra

Department of Mathematics, Statistics and Computer Science

College of Basic Sciences and Humanities

Govind Ballabh Pant University of Agriculture and Technology

PANTNAGAR, INDIA

E-MAIL: MSSHAS@CITYU.EDU.HK 